\title{
EXPERIENCES IN THE STUDY OF PORTAL HYPERTENSION
}

\author{
A. K. Basu, M.S.(Cal.), F.R.C.S.(Eng.), F.A.C.S. \\ Director-Professor, Department of Surgery, Institute of Postgraduate Medical Education and Research, Calcutta
}

THE pathogenesis, clinical features and the management of cases of portal hypertension have aroused widespread interest. Recent advances in radiological and biochemical methods of investigation have brought to light some important aspects of this problem. The clinician can at present obtain a fairly accurate idea of the anatomical or even the pathological abnormalities that are present in a particular case even prior to operation. The behaviour pattern of many cases can be predicted with or without operation with reasonable certainty. However, in some areas, the syndrome still remains obscure.

\section{Material and Methods}

In the course of the last eight years, we have had the opportunity of looking after and operating on over 100 cases of portal hypertension of greater or lesser degree. Sixty-two of these cases have been investigated in great detail and operated on at the S.S.K.M. Hospital during the last five years and these form the main basis of this study. A record of our earlier cases has been published elsewhere (Basu, Das and Aikat, 1957). Many new and interesting facts have come to our notice during the course of this investigation and new knowledge has been gained.

In addition to careful scrutiny of their past history with particular regard to protozoal infections, jaundice, haematemesis, ascites, etc., and examination of their physical state, special investigations were carried out on the following lines: (I) Haematological - examination of the peripheral blood and bone marrow study. (2) Biochemicalexamination of plasma proteins and liver function tests. (3) Radiological—oesophagogram and splenoportal venogram.

Opportunity was taken at the time of operation to investigate the following:

(a) Estimation of the portal venous pressure.

(b) Macroscopic appearances of the spleen, liver and of the portal venous system including presence of collateral venous channels.

(c) Examination of biopsy material from the liver, taken at operation.
Cases have been followed up clinically, and $\vec{\omega}$ further haematological and radiological investigations have been carried out during the follow-up period. Detailed liver function tests have been performed. The histology of the liver has been. studied by repeated liver biopsy.

\section{Results}

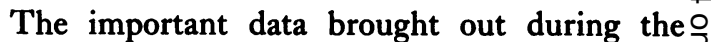
course of this study are presented in the tables, Table I summarizing the observations made before $?$

TABLE I

ANALysis of ObSERVATIONS Before Operation AND Evaluation of Portal Hypertension

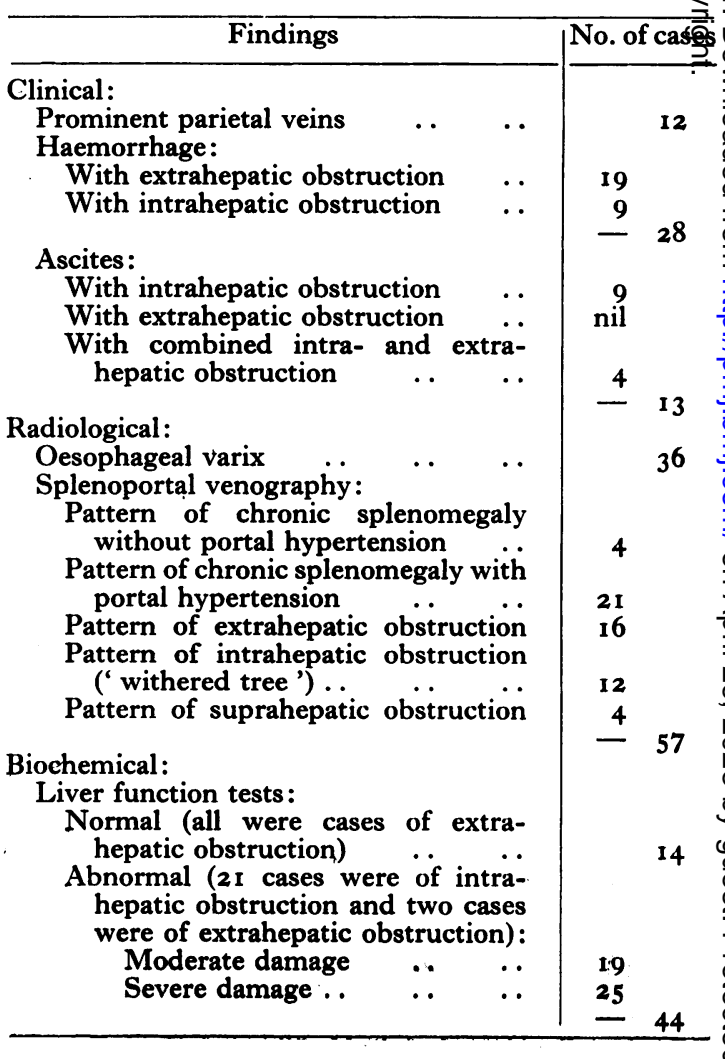




\section{TABle 2}

ANalysis OF THE ObServations MADE AT Operation under DiffERENT HeadS

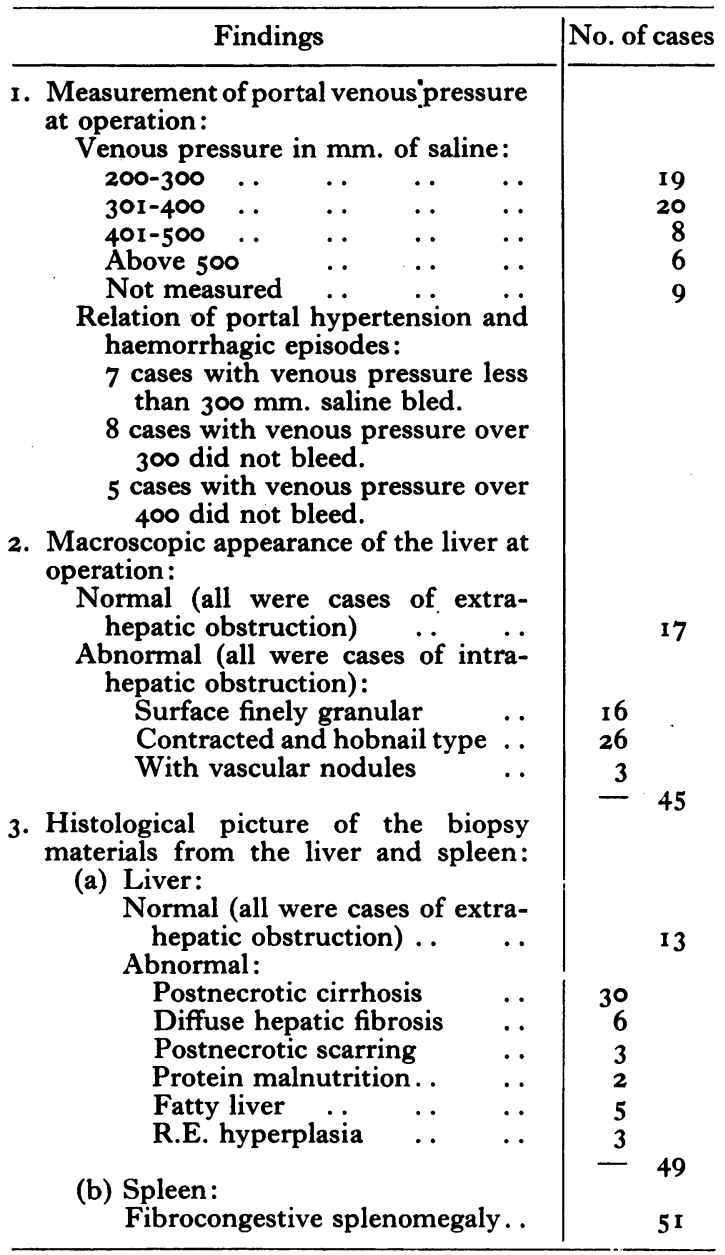

operation, and Table 2, those made at operation. The types of block giving rise to the portal hypertension are listed in Table 3 .

\section{Types of Operation Performed}

Splenectomy only was done in 22 cases. Splenectomy with spleno-renal shunt in 16 cases, portacaval shunt in 18 , ligation of hepatic and splenic artery in three and ligation of splenic artery alone in one case.

\section{Overall Follow-up of Cases}

Fifty-six cases have been followed up for periods varying from six months to five years. Clinically 45 cases are doing well, seven cases had recurrence of haemorrhage and four cases have developed ascites.
TABLE 3

Types of Block Causing Portal Hypertension

\begin{tabular}{|c|c|c|}
\hline Type of block & & No. of cases \\
\hline $\begin{array}{l}\text { I. Extrahepatic obstruction: } \\
\text { Pancreatic tumour... } \\
\text { Pancreatitis with fibrosis } \\
\text { Portal vein thrombosis } \\
\text { Cavernomatous transform } \\
\text { vein ... .. }\end{array}$ & 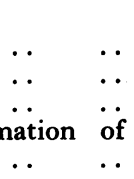 & $\begin{array}{l}4 \\
9 \\
3 \\
4\end{array}$ \\
\hline $\begin{array}{l}\text { 2. Intrahepatic obstruction } \\
\text { 3. Combined intra- and ext } \\
\text { obstruction: } \\
\text { Cirrhosis plus hyperplastic } \\
\text { Cirrhosis plus portal vei } \\
\text { bosis .. . . }\end{array}$ & $\begin{array}{l}\text { ra-hepatic } \\
\text { nodule.. } \\
\text { in throm- } \\
\ldots \quad . .\end{array}$ & $\begin{array}{ll}- & 36 \\
2 & \\
2 & \\
2 & \end{array}$ \\
\hline $\begin{array}{l}\text { 4. Suprahepatic obstruction: } \\
\text { Polycythaemia vera } \\
\text { Constrictive pericarditis }\end{array}$ & 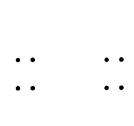 & $\begin{array}{l}\mathbf{I} \\
\mathbf{I} \\
-\end{array}$ \\
\hline
\end{tabular}

Repeated haematology studies were done in 28 cases; 26 showed improvement as compared to pre-operative status. Out of 24 cases in which repeated liver function studies were done, 18 cases showed improvement as compared to pre-operative status. Repeated liver biopsy studies were done in 14 cases out of which nine cases showed improvement.

\section{Results of Extrahepatic Obstruction Cases and of Shunt Operations}

Out of 20 cases of extrahepatic obstruction, 18 were operated on. Two cases died before operation due to severe haemorrhage; two cases died after operation; one case of portal vein thrombosis died seven days after operation; and one case of pancreatic tumour died four days after operation. The remaining 16 cases are doing well.

Spleno-renal shunt was performed in 16 cases. The mortality was nil. Recurrence of haemorrhage occurred in three cases. Thirteen cases are doing well (six months to four years).

Porta-caval shunt was done in 18 cases. One had recurrence of haemorrhage.

\section{Discussion}

It will be seen that next to intrahepatic obstruction due to cirrhosis of liver, the infrahepatic obstruction cases form the second largest group in our series (Table 3 ). The importance of this mode of obstruction in the pathogenesis of portal hypertension has been realized in recent years (Das and Basu, 1956). It is fortunate that surgical treatment in this group can in many cases be curative. This is in contrast to intrahepatic or even suprahepatic obstruction, where treatment at 


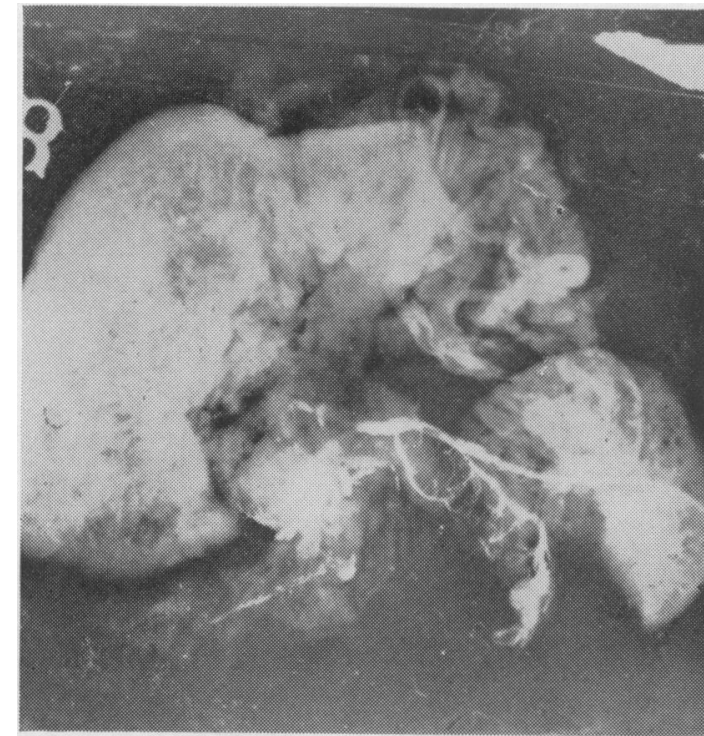

FIG. I.-Liver, spleen and pancreas showing obstruction of the splenic vein by pancreatic fibrosis.

best is only palliative and helps in prevention of haemorrhage. Of the 18 cases in our group who were operated on, 16 are doing well, are free from haemorrhage and doing full time work. In three of these cases, the follow-up period has exceeded four years.

The causes of infrahepatic obstruction of the portal venous system may be of different types. We were fortunate to meet with nearly all these possible types. The largest number (nine cases) were due to the splenic vein being enveloped by fibrosis associated with chronic fibrosing pancreatitis. Eight of these cases were observed at operation and in the ninth case who unfortunately succumbed to a severe episode of haemorrhage prior to operation, we were able to do an autopsy study and demonstrated the obstruction by passing a fine tube in the splenic vein which got obstructed at the site of fibrosis; radio-opaque dye introduced through this tube did not pass beyond this site (Fig. I).

We had four cases of splenic vein obstruction due to tumour of the pancreas. In one case, it was possible to do a partial pancreatectomy together with splenectomy and ligation and division of the large tortuous veins draining towards the fundus of the stomach. The venogram (Fig. 2) of the patient was highly interesting.

There were three cases of portal vein thrombosis producing hypertension. All were confirmed at operation. One case was associated with advanced cirrhosis of liver. This case died seven days after operation due to progressive hepatic failure and

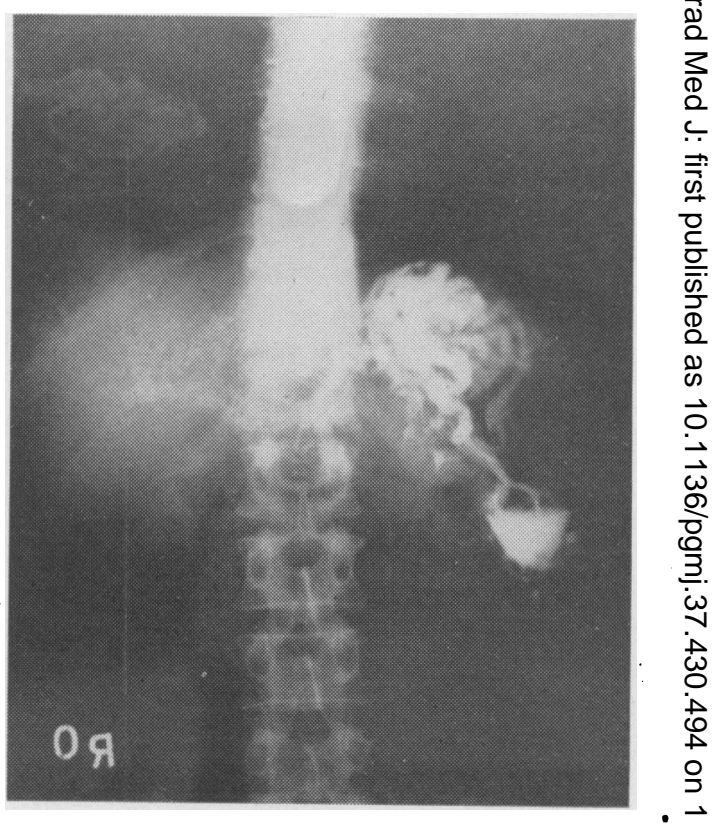

FIG. 2.-Spleno-portal venogram showing obstruction of the splenic vein by tumour of the pancreas and numerous collaterals directed to the fundus of the $\frac{\bar{s}}{2}$ stomach.

dissection of the autopsy material demonstrated large linear firm thrombus extending from the trunk of the portal vein and occluding the left branch nearly completely. This also explained the marked atrophy of the left lobe in this case.

We have had at least four cases of cavernomatous $\stackrel{\square}{\square}$ transformation of the portal vein. Instead of the $\overrightarrow{\overrightarrow{0}}$ normal large portal vein, there are multiple 3 tortuous tributaries of smaller calibre enveloping the right border of the lesser omentum (Fig. 3). We were able to demonstrate three of these cases by venography and confirmed the abnormal anatomy at operation.

Another was, however, not shown by venography but was observed during the attempt to do a porta-caval shunt. Small cavernomatous tribu-

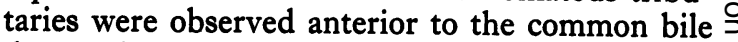
duct and the portal vein was absent from its usual $\frac{D}{O}$ place posteriorly. It is to be expected that the liver is likely to be normal in all cases of extra- $N$ hepatic obstruction group. This was exemplified in our series both on macroscopic appearance at $\mathrm{N}$ operation and also on histology.

The intrahepatic obstruction group in our series was all due to cirrhosis of the liver. It is conceivable that obstruction to the portal venous $\stackrel{D}{\mathscr{D}}$ stream within the liver may also be produced by other pathological conditions such as tumours, $\frac{0}{0}$ cysts or abscesses, but these instances must be $\underset{\mathbb{D}}{ }$ extremely uncommon. The common histological $\frac{\stackrel{9}{\mathbb{D}}}{\sigma}$ 


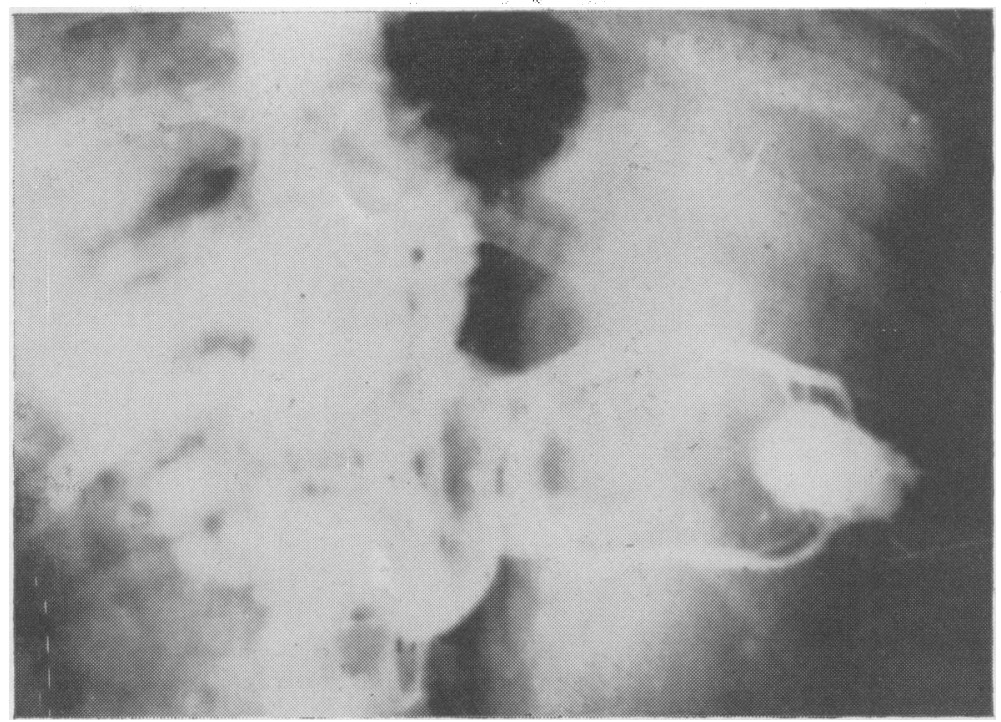

FIG. 3.-Spleno-portal venogram showing cavernomatous transformation of portal vein.

type of cirrhosis met with was postnecrotic cirrhosis with nodular hyperplasia. This type of histology was also evident in the macroscopic appearance of large relatively soft dark looking bosses on the surface of the liver of ten seen at operation.

We have had four cases in which intra- and extrahepatic obstruction were both responsible for portal hypertension (Table 3). Two cases were due to a large hyperplastic liver regeneration nodule of the type just described and in the other two, portal vein thrombosis was apparently secondary to advanced cirrhosis of the liver.

The syndrome of suprahepatic obstruction or obstruction to the outflow of the portal venous blood from the liver has only recently been described. The so-called Budd-Chiari syndrome in which there is thrombosis of the minute hepatic venous tributaries before they join the inferior vena cava is an example of this syndrome. This type of obstruction causes severe degrees of portal hypertension, sometimes combined with ascites in later stages. There were two cases in our series in which we decided that this particular type of obstruction was the responsible factor for portal hypertension (Table 3). One was a case of polycythaemia vera, who demonstrated the pattern of venogram which we have associated with suprahepatic obstruction and in which the histology of the liver biopsy material showed evidences of hepatic venous tributary occlusion. The other was a confirmed case of constrictive pericarditis who also had the typical venographic patterns and in whom pericardectomy relieved the portal hypertension. It would be well to realize that this type of obstruction though relatively rare can be diagnosed fairly accurately by careful venographic or histologic studies prior to exploration. It should also be remembered that in the later stages of this type, the liver may develop advanced secondary cirrhotic features and then the syndrome would be indistinguishable from the intrahepatic obstruction group.

We tried to ascertain the relative values of the different methods of investigation-clinical, biochemical and radiological in the pre-operative evaluation of the presence, the degree and the type of portal hypertension. From the data available, it would seem that each of these methods of investigation is of importance but perhaps the most important is the study of the living circulatory anatomy by spleno-portal venography. The important points in the clinical signs and symptoms as brought out in our series are as follows:

(I) The visual evidence of prominent parietal veins is a late and therefore uncommon sign of portal hypertension. It was present in only 12 of our cases (Table $\mathrm{I}$ ).

(2) Haemorrhage is a more common feature of extrahepatic obstruction group than of intrahepatic obstruction (Table I). It is also more severe and therefore sometimes fatal in the former group. Nearly all our cases of extrahepatic obstruction had history of severe and repeated haematemesis and two cases were fatal before operation. On the other hand, only nine of the 36 intrahepatic obstruction group had history of lesser degrees of haemorrhage.

(3) As opposed to this, ascites seldom occurs in extrahepatic obstruction whereas it was present in 13 cases of our intrahepatic obstruction group (Table I). 


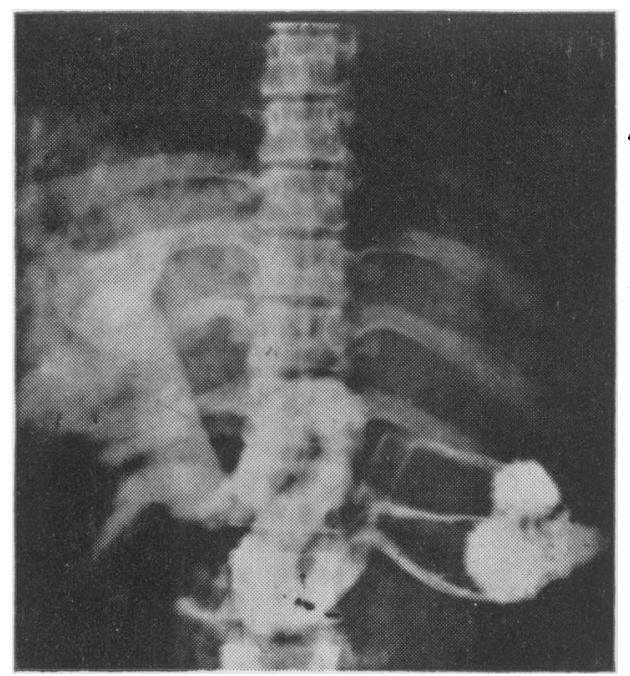

FIG. 4.-Pattern of spleno-portal venography in chronic splenomegaly without portal hypertension.

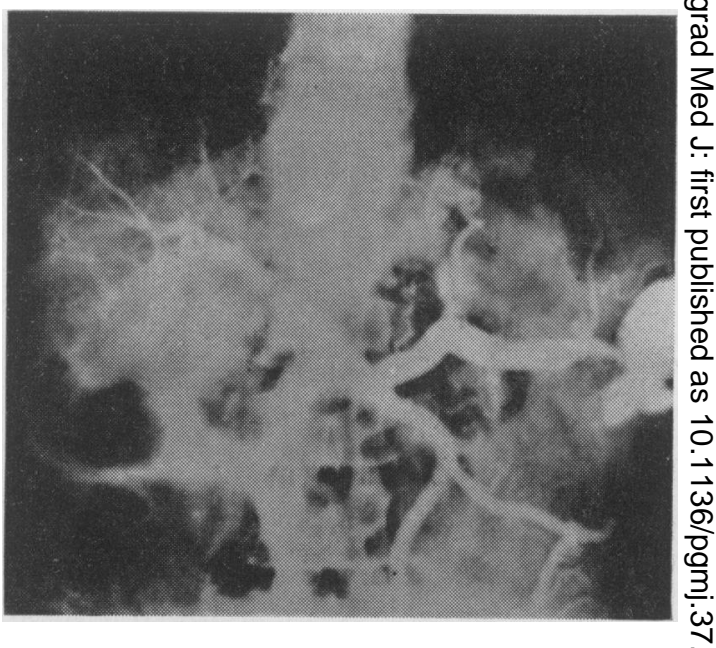

FIG. 5.-Pattern of spleno-portal venography in chronicit splenomegaly with cirrhosis of liver and portalo hypertension. Collaterals shown.

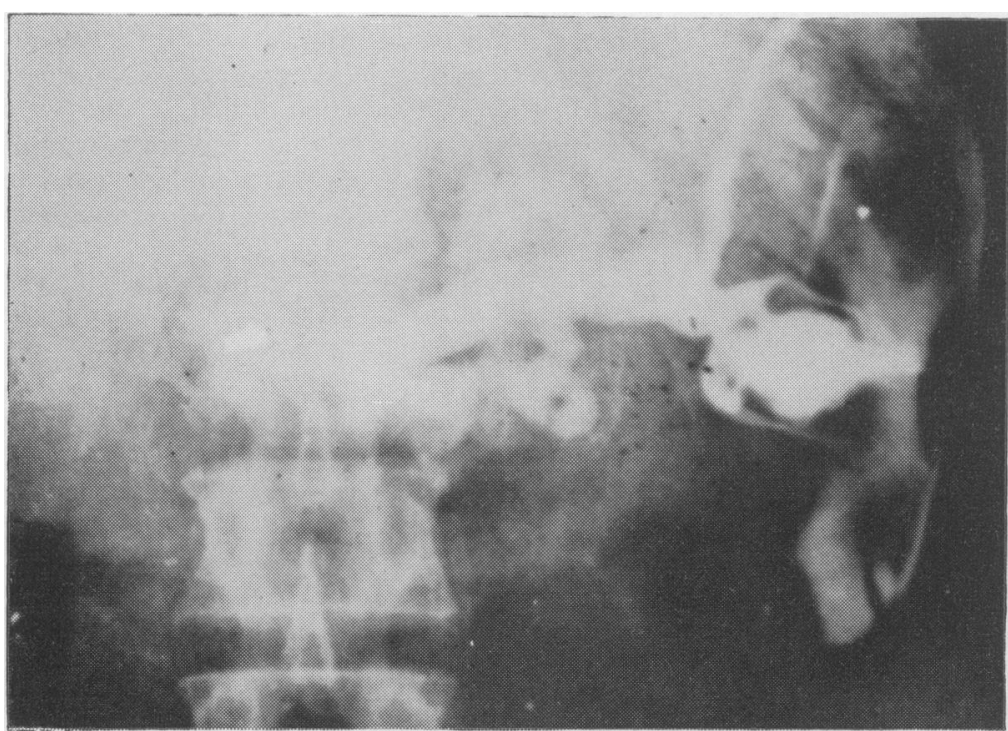

Fig. 6.-Pattern of chronic splenomegaly with infrahepatic obstruction of the splenic afdo portal vein with collaterals.

The biochemical tests for liver function are individually well known for their incompleteness. One has to rely on a battery of tests to obtain an overall picture of liver function. In addition to the usual tests, we performed a careful electrophoretic study of the globulin fractions of the serum and this, we believe, gives a good idea of the state of liver function. When the total serum protein was less than $5 \mathrm{~g}$. $\%$ and the serum albumin less than $2.5 \mathrm{~g}$. $\%$, when there was reversal of albumin and globulin ratio and when particularly serum electrophoresis showed hyperglobulinaemic peak in the globulin fraction, we considered the liver function to be markedly deranged. On this basis, we considered that ino 44 of our cases, the liver was functionally moder-? ately to severely damaged. It would be seen thato these were all cases of the intrahepatic obstruction ${ }_{N}$ group and that in extrahepatic obstruction, livero function, as is to be expected, remains relatively $N_{0}$ normal.

The importance and value of spleno-portal $\omega$ venography in the diagnosis and elucidation of portal hypertension has been described by us in $\frac{\mathbb{\Phi}}{\complement}$ detail elsewhere (Basu and Das, 1956).

We would therefore only point out and em- 0 phasize that this method of investigation is simple,

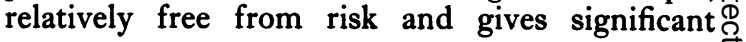




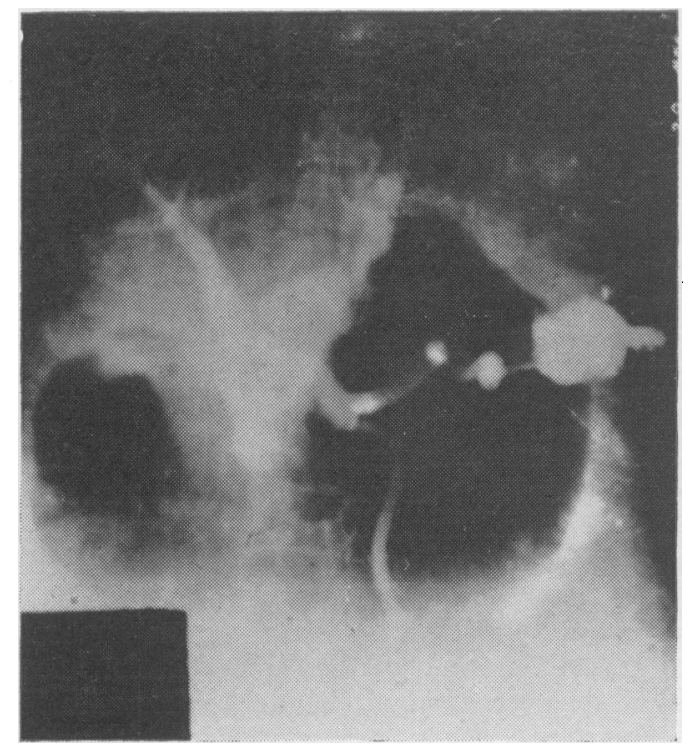

FIG. 7.-Pattern of spleno-portal venography in cirrhosis of liver without splenomegaly. 'Withered tree' appearance of the intrahepatic radicles. Collaterals are numerous.

information with regard to the type and the site of obstruction and the anatomical abnormality of the vascular pattern is well delineated (Table I). With some experience in the study of the relevant venography patterns, even the nature of the obstructive agent can be predicted with fair degree of accuracy. We have described five distinct venographic patterns which it would be worthwhile to recapitulate here.

(I) Pattern in chronic splenomegaly without portal hypertension-dilated tortuous splenic and portal vein without collaterals. Intrahepatic radicles faint (Fig. 4).

(2) Pattern in chronic splenomegaly with cirrhosis of liver and portal hypertension-same as above but with collaterals (Fig. 5).

(3) Pattern of infrahepatic obstruction-obstruction in the course of the splenic or portal vein with collaterals (Fig. 6).

(4) Pattern in intrahepatic obstruction (cirrhosis of liver)-splenic and portal vein not dilated or tortuous; collaterals present; intrahepatic radicles distorted and truncated. "Withered tree' appearance. Collaterals prominent (Fig. 7).

(5) Pattern in suprahepatic obstruction-splenic vein and portal vein not dilated but collaterals present. Intrahepatic radicles well seen right up to periphery (Fig. 8).

In our experience, demonstration of collaterals in splenoportal venography is an accurate guide to the presence as well as to the degree of portal hypertension and is seen earlier than other indications such as haematemesis, ascites or deranged liver function tests.

We also believe that spleno-portal venography is of great help to the surgeon in the planning of the requisite operation.

As a routine measure, we measured the portal venous pressure at operation by a saline manometer. In many cases, the intra-splenic pressure was measured in addition. In the majority of our cases, the range of pressure varied between 200 and $400 \mathrm{~mm}$. saline. There were eight cases with pressures above $400 \mathrm{~mm}$. and six cases above 500 mm. (Table 2).

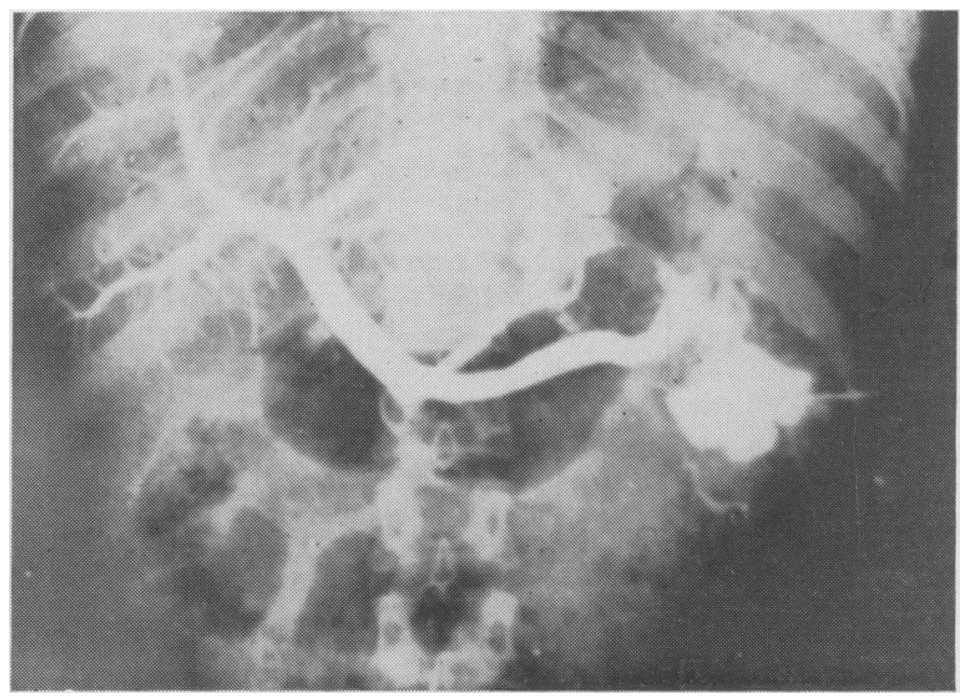

Fig. 8.-Pattern of spleno-portal venography in suprahepatic obstruction. Splenic and portal veins are not dilated. Collaterals are marked. 
It appeared from a study of our cases that there was no definite relation between the incidence of haemorrhage and the degree of portal hypertension. Seven cases of less than $300 \mathrm{~mm}$. saline pressure had severe haemorrhage whereas eight cases above $300 \mathrm{~mm}$. and five cases above $400 \mathrm{~mm}$. pressure did not bleed. It appears therefore that the incidence of haemorrhage in portal hypertension depends more on the anatomical distribution of the abnormal collaterals that are formed as the result of the varied site of obstruction rather than on the absolute measure of the portal venous pressure.

The plan of our operative management was decided upon as follows. Most of our cases had very large spleens with evidence of advanced hypersplenism. Splenectomy therefore was considered essential in many of the cases. In addition, we decided to do a shunt operation specifically in those cases where there was history of significant haemorrhage.

The specific role of splenectomy alone in the management of portal hypertension has been debated and it has been stated that the surgeon, who in cases of portal hypertension is doing a splenectomy alone and not combining it with a shunt procedure, is not doing the best possible thing for his patient. That is probably true in the type of cases where large splenomegaly is not the most important factor and where there are evidences of repeated haemorrhages. In our country, we come across a large number of cases where chronic splenomegaly is associated with secondary hepatic fibrosis and where instead of repeated haemorrhages, clinical evidence of hypersplenism is the most important factor. The evidence of portal hypertension in such cases is the enhanced portal tension as measured at operation. In such types of cases, we have contented ourselves by doing splenectomy alone and have been carefully following our cases with repeated haematological, liver function and liver biopsy studies. Until now, only one such post-splenectomy case has come back with haemorrhage. The value of splenectomy in this group of cases lies in the cutting off of the large volume of splenic effluent and therefore of the large portal hypervolaemia and along with this, the factor of hypersplenism is done away with. We have made some efforts in the volumetric determination of the actual amount of splenic effluent in these cases by the Evans blue dye method. Our preliminary studies show that in large splenomegalies, this amount at least exceeds 600 c.c. per minute.

For many cases of extrahepatic obstruction, splenectomy with ligation and division of abnormal collateral vessels is all that is necessary and is curative.
Where history of haemorrhage is significant, $a_{\varrho}$ shunt procedure is imperative and we have per- $z$ formed this on 34 occasions- 16 spleno-renal and $\stackrel{\mathbb{Q}}{\varrho}$ I 8 porta-caval. We maintain that although porta-..caval shunt is a technically easier procedure, $\underset{\vec{*}}{\vec{*}}$ spleno-renal shunt with splenectomy is the opera- $\frac{\text { f }}{9}$ tion of choice in many of the cases we meet with. 등 We would reserve porta-caval procedure for the $\frac{\overline{\bar{c}}}{\bar{D}}$. type of cases where splenomegaly is not significant $\frac{\bar{\sigma}}{\bar{\sigma}}$ but where there is a history of repeated haemor- $\stackrel{\mathbb{Q}}{\Omega}$ rhages and also for the post-splenectomy or for the post-spleno-renal shunt bleeder.

The operative approach either to the shunt $\stackrel{\overrightarrow{ }}{\overrightarrow{0}}$ operation or even to splenectomy in the large $\vec{\sigma}^{-}$ splenomegalies is now a standardized procedure in our hands. There is no question that abdomino- -3 . thoracic approach combined with division of the diaphragm gives incomparably better exposure ${ }_{t}^{-}$ and ensures division of the large adhesions con- $\overrightarrow{0}$ taining tortuous vascular channels either with the undersurface of the diaphragm or within the $\$$ posterior layer of the lienorenal ligament with precision and deliberation. Since we started using this approach regularly about four years ago, we $\rightleftarrows$ have never had cause to regret it.

In addition to regular clinical check-up, we have $\stackrel{\frac{5}{9}}{\rightarrow}$ been doing repeated haematological, liver function $\vec{\theta}$ and liver biopsy studies of our cases - at three or

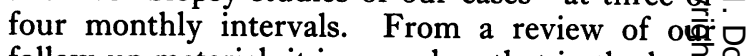
follow-up material, it is now clear that in the large majority of our cases, the operation has been well worthwhile. Of the total of 56 cases followed up from six months to five years, 45 cases are clinically $\frac{2}{\mathbb{Q}}$ doing well, only seven have had recurrence of $\stackrel{\varrho}{\vec{T}}$ haemorrhage and four have developed ascites. $\frac{\hat{3}}{3}$ Liver function and liver biopsy studies show improvement in a good proportion. The haematological status has markedly improved indicating elimination of the factor of hypersplenism. We 3 . have been trying to analyse the effect of splenectomy on the histology of the liver in those cases where cirrhosis was already observed at operation. 8 It is yet early to state our opinion categorically but we have been impressed by positive functional as 0 well as structural improvement in at least some of our cases indicating the reversibility of the fibrotic process in the liver and the injurious influence of the enlarged spleen on the liver parenchyma.

In extrahepatic obstruction, as is to be expected, $N$ the results of operations are even better. Except $\underset{\omega}{\omega}$ for the two post-operative deaths, all the remaining 16 are doing well and to all appearances are cured. $\bullet$ Of 16 spleno-renal shunts, there has been recurrence of haemorrhage in three cases. The other $\stackrel{?}{?}$ I 3 cases are doing well and are free from haemor- $\frac{7}{0}$ rhage. In two cases, the follow-up period has exceeded four and a half years. 
A few significant observations made as the result of this study may be summarized:

(1) Haemorrhagic episodes are more common with extrahepatic than with intrahepatic obstruction, whereas ascites seldom occurs with extrahepatic obstruction.

(2) The most important diagnostic agent is spleno-portal venography which is simple, relatively free from risk and gives significant information with regard to the site, and also sometimes the nature of the obstructive agent.

(3) Electrophoretic study of serum proteins and protein fractions are the best guide to the state of liver function.

(4) There is no definite relation between portal hypertension and the incidence of haemorrhagic manifestations.

(5) In the pathogenesis of portal hypertension, the most common obstructive agent is intrahepatic obstruction due to cirrhosis of the liver. However, infrahepatic obstruction due to different causes comes a close second. Suprahepatic obstruction though rare is sometimes responsible.

(6) For cases associated with repeated haemorrhage, splenectomy associated with spleno-renal shunt is the operation of choice in the types of cases we meet with. Porta-caval shunt is indicated where splenomegaly and hypersplenism are not significant or for the postsplenectomy or the postspleno-renal bleeder. Simple splenectomy is indicated where haemorrhagic episodes are absent.

(7) The effect of the operation in relation to the hepatic function and structure and the general nutritional and the haemopoietic status of the patient has been studied by a careful follow-up programme. There is evidence to show that in many cases there is reversal of the fibrotic process in the liver and sustained improvement of liver function after this operation.

(8) Infrahepatic obstruction cases are markedly benefited and can be considered as cured by the operation.

\section{Summary}

This study presents a detailed review of cases of portal hypertension dealt with during the last five years. Earlier cases already reported on have been omitted from the study except for general observations.

The review includes careful evaluation of the methods of diagnosis and the importance of the various diagnostic agents. A study is made of the relation of portal hypertension to the haemorrhagic manifestations. The role of different types of obstruction and the nature of the obstructive factors in the pathogenesis of portal hypertension are clearly brought out. The indications of the different types of operations and the results of such operations are discussed.

I am grateful to Prof. Aikat for his interest in this investigation and for all the help in the liver function, hæmatological and liver biopsy studies. Thanks are also due to the Superintendent, S.S.K.M. Hospital for the use of the hospital records.

\section{REFERENCES}

BASU, A. K., DAS, A., and Aikat, B. K. (1957): Observations on Fifty Operated Cases of Chronic Splenomegaly,

F. Indian med. Ass., 28, 380 .
and DAS, A. (1956): Splenoportal Venography for Evaluating Abnormalities of Portal Circulation, Brit. med. $\mathcal{~}$., ii, 916.

Das, A., and Basu, A. K. (1956): Portal Hypertension Due to Extrahepatic Obstruction, Ibid., i, 325. 